\title{
Pattern Width Homogenization in the Field of Liquid-Crystal-Display Matrix Exposure by Adjusting Each Cell Transmittance
}

\author{
Toshiyuki Horiuchi, Shota Haneishi, Yumika Yoshida, and Hiroshi Kobayashi* \\ *Department of Mechanical Engineering \\ Tokyo Denki University, \\ 5 Senju-Asahi-cho, Chiyoda-ku, Tokyo 152-8552, Japan
}

\begin{abstract}
Width homogenization of patterns printed by the desktop matrix-exposure system using a commercial liquid-crystal-display projector was investigated. At first, it was thought that the pattern width distribution caused by the dispersion of illumination-light intensity would be easily compensated by adjusting each bright cell transmittance. However, it was fundamentally difficult. Bottom peaks of the image intensity did not reach zero caused by the diffraction and intrinsic transmittance of black cells. For this reason, the bottom peaks also distributed depending on the illumination-light intensity. As a result, image contrast was degraded by reducing the transmittance of bright cells. To print line-and-space (L\&S) patterns homogeneously in the exposure field, it was also necessary to increase the transmittance of black cells at places where the illumination-light intensity was low. By this countermeasure, down to 2-pixel L\&S patterns were printed almost homogeneously. The width distribution of 4and 5-pixel L\&S patterns was suppressed almost within $\pm 10 \%$.

Keywords: matrix exposure, pattern width homogenization, intensity distribution, liquid crystal display, image contrast
\end{abstract}

\section{Introduction}

Lithography is mainly used for printing extremely minute patterns of highly integrated semiconductor devices. However, it is also widely applied to patterning of large patterns with widths or sizes of 10-100 $\mu \mathrm{m}$ used for fabricating various sensors, fluidic devices, coils, lenses, and others.

It is impractical to apply a lithography system or an exposure system for the use of semiconductor device fabrication to fabrication of above mentioned micro-parts. In the case of exposure system for the use of semiconductor device fabrication, the resolution or the minimum printable pattern size is the most important specification of the system. For this reason, projection optics with a very high numerical aperture is used, and the depth of focus (DOF) is very shallow. Accordingly, the high-resolution patterning performance is obtainable only when patterns are printed on extremely flat substrates under the focusing conditions that the pattern images are clearly formed within the shallow DOF range.

However, in the case of large-pattern printing for the use of micro-parts, substrates or objects to be exposed are not usually as flat as silicon wafers. In addition, required resist thicknesses are roughly correspondent with the pattern width. Accordingly, the DOF of the exposure system has to be sufficiently larger than the flatness errors of the substrate surface plus the resist thickness coated on the substrate. Therefore, the numerical aperture of the projection optics has to be far smaller than that of exposure optics used for semiconductor device fabrication. Besides, in the fabrication of such micro-parts and micro-systems, numbers of products are not generally large as those of the semiconductor devices such as memories. On the other hand, 
various kinds and types of products are often required.

For this reason, costs of masks or reticles used for lithography often affect the product costs. Therefore, maskless lithography systems are preferred, and various exposure systems using liquid-crystal-display (LCD) panels in place of reticles have been proposed [1-7]. The authors also developed several systems in the past researches [8-14], and recently, proposed a very low-cost exposure system using a commercial LCD projector as it was [15].

In the new LCD exposure system, the magnification projection optics attached originally in the projector was removed, and a camera lens for macro-photography (Sigma, 50mm F2.8 EX DG MACRO) was attached instead. However, the original projector did not have an alignment mechanism of the lamp-source position. In addition, because the camera lens was placed in the frame cover of the projector, it was difficult to adjust the lens to the best position for homogenizing the light intensity. Therefore, image intensity of patterns was much distributed, and it was difficult to print patterns homogeneously in the exposure field. For this reason, a new measure to improve the patterning homogeneity was investigated here.

\section{Desk-top Exposure System}

The desktop exposure system using a commercial LCD projector (Mitsubishi Electric, LVP-HC5000) was used for the research. The system was developed in the past research, and the outside view of the system is shown in Fig. 1. The projector was equipped with three LCD panels of red, green, and blue. The cell size of the panels was $7.4 \mu \mathrm{m}$ square, and the width of the black matrix lines was $1.1 \mu \mathrm{m}$. Accordingly, the cell pitches of the LCD panels were $8.5 \mu \mathrm{m}$ in X and Y directions. The pixel number of each LCD panel was $1,920 \times 1,080=2,073,600$. However, in the past research, the pixel number of the note-type personal computer used for assigning patterns on the projector LCD was $1,024 \times 768=786,432$. For this reason, the projection ratio was changed, and the minimum half pitch of printed line-and-space patterns was $14.1 \mu \mathrm{m}$. To improve the element pattern pitch by adjusting the pixel numbers of LCD panels of the projector and the computer, the personal computer used for assigning the patterns

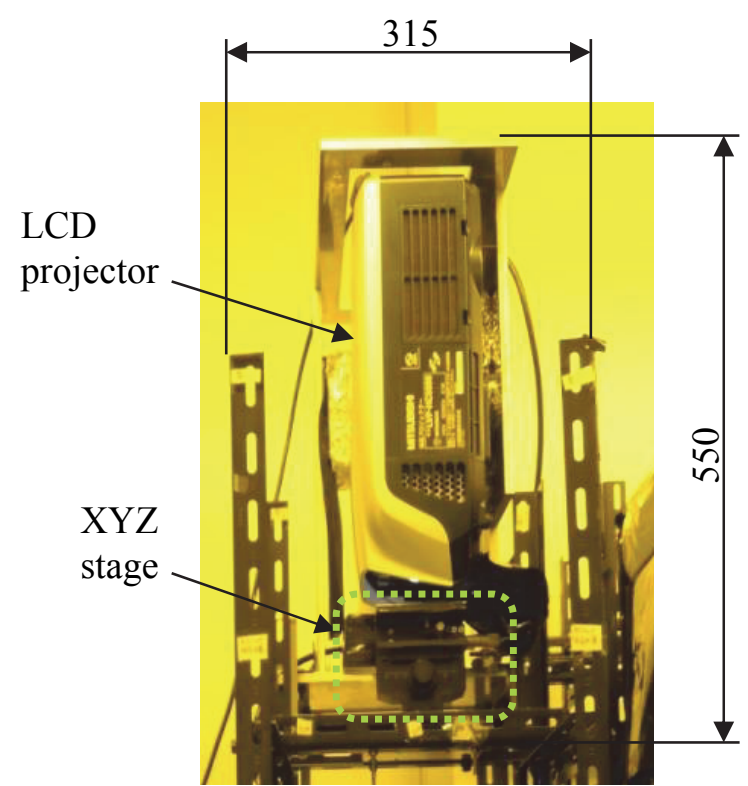

Fig. 1. Desktop exposure system developed in the past research using a commercial LCD projector.

was changed to the new one (NEC, PCGN174Z2A3). As a result, the minimum element pattern pitch was reduced to $9.3 \mu \mathrm{m}$, and the exposure field size was improved from approximately $13 \times 10 \mathrm{~mm}^{2}$ to $17.5 \times 10 \mathrm{~mm}^{2}$.

It is preferable that the pattern widths are larger than two pixel sizes for the matrix exposure using LCD panels, because the printed pattern width and position become controllable by changing the transmittances of neighbored cells [11]. In addition, minute notches or bulges caused by the black matrix appear at the side walls of patterns, if the resolution of the optics is roughly higher than the pixel pitch. For these reasons, the $\mathrm{F}$ number of the camera lens was set at $F=16$.

The actual projection ratio of the lens was $\mathrm{m}=9.3 / 8.5=1.09$. Accordingly, the effective $\mathrm{F}$-number $\mathrm{Fe}$ of the lens was

$$
F e=(1+m) F=33.4 \text {. }
$$

The numerical aperture NA was

$$
N A=\frac{1}{2 F e}=\frac{1}{2 \times 33.4}=0.015 \text {. }
$$

Therefore, the resolution limit $R$ was roughly estimated as

$$
R=k_{1} \frac{\lambda}{N A}=0.5 \times \frac{0.436}{0.015}=14.5(\mu \mathrm{m}) .
$$

Here, $\lambda$ was assumed to be $0.436 \mu \mathrm{m}$ judging from the spectral light-intensity curve measured actually using a spectrometer (Ocean Optics, HR 4000), as shown in Fig. 2. The light source of the 


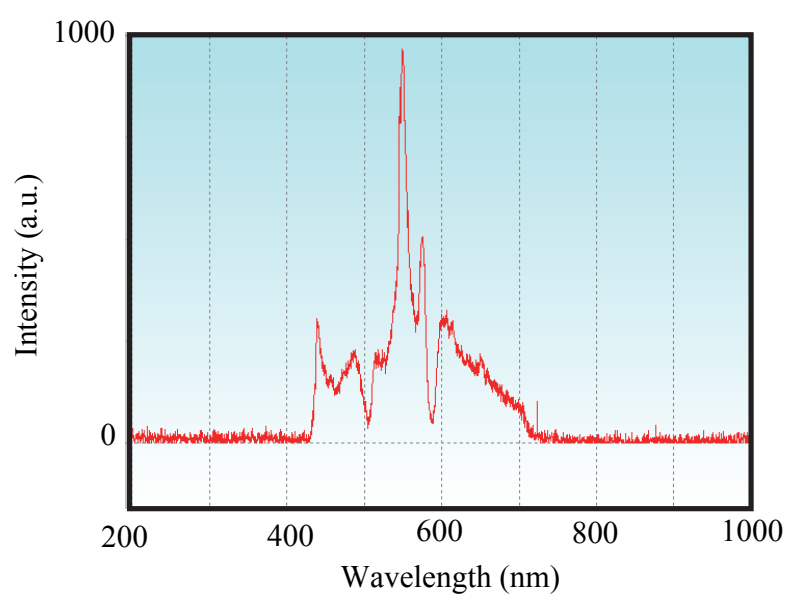

Fig. 2. Measured spectral light-intensity curve.

projector was a high-pressure mercury lamp with power of $160 \mathrm{~W}$.

The light intensity distribution in the exposure field was measured using a detector (Ushio, UIT-250), as shown in Fig. 3. It was clarified that the light intensity was weakest at the upper left corner, and the minimum intensity was approximately a half of the maximum one. This disparity was probably caused by the positional adjustment errors of the light source and the projection lens.

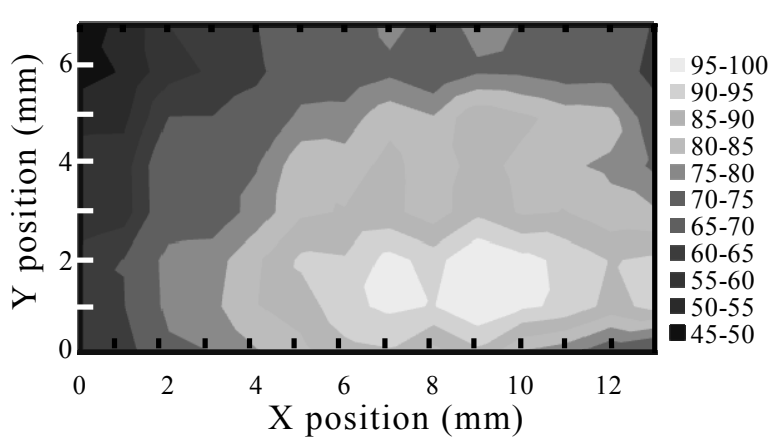

Fig. 3. Light intensity distribution in the exposure field when all the cells are assigned to white.

\section{Improvement of Homogeneity}

Because no means were prepared to adjust each position, it was tried to improve the severe distribution by individually controlling the transmittance of LCD cells and homogenize the patterning performances in the exposure field. L\&S patterns with widths of 2-5 pixels were used as the test patterns. Horizontal L\&S patterns were laid in the whole exposure field, and the evaluation area was divided into 32 parts composed of 4 lines and 8 columns. As a resist, positive OFPR 800 (Tokyo Ohka Kogyo) coated in $1 \mu \mathrm{m}$ thick was used. This is a typical g-line resist appropriately sensitive to light with wavelengths around $436 \mathrm{~nm}$.

At first, it was thought that the light intensity disparity would be easily modified by changing the transmittance of each liquid crystal cell in inverse proportion to the light intensity. For this reason, each transmittance of bright liquid-crystal cell was reduced in proportion to the measured light intensity, as shown in Fig. 4. However, the patterns were not homogeneously printed at all, as shown in Fig. 5. In spite of reducing the transmittance of bright cells of 2-pixel L\&S patterns, exposure light was still too strong at the right bottom. On the other hand, light intensity was still weak around the upper left corner. It was obvious that the imbalance was still terrible.

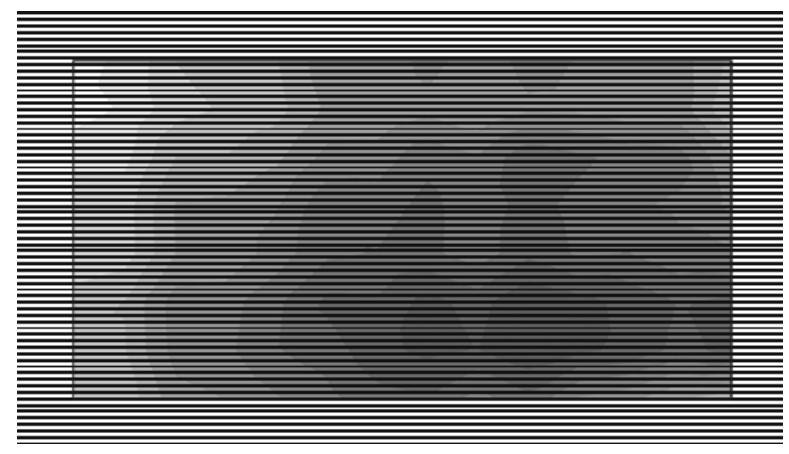

Fig. 4. Patterns designed for compensating the intensity distribution and superimposed on L\&S patterns. The transmittance was compensated in the rectangular area in the center.

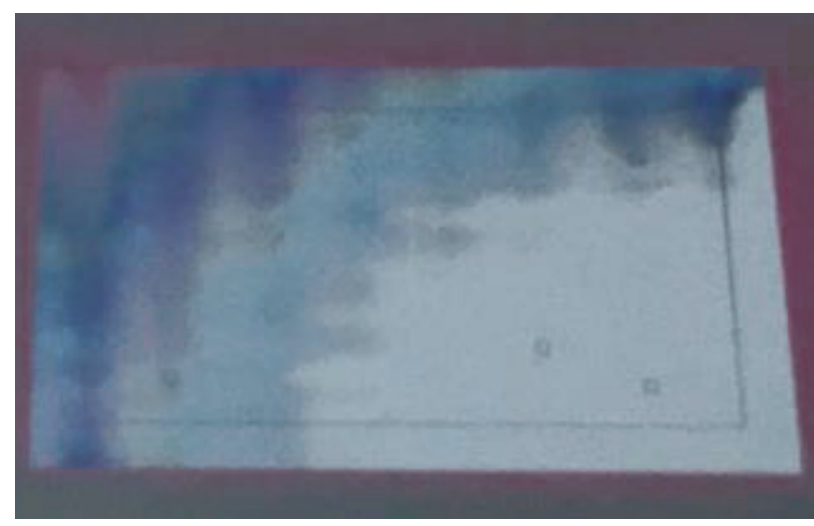

Fig. 5. 2-pixel L\&S patterns printed by adjusting only the bright cell transmittance using the compensation patterns shown in Fig. 4. The exposure disparity was not sufficiently improved, and the imbalance was still terrible. 
Therefore, reasons why the patterns were not homogeneously printed were deliberated. As a result, the reasons were assumed as follows.

The widths of 2-pixel L\&S patterns were quite near to the resolution limit of the projection optics. For this reason, it is assumed that bottom peaks of the pattern-image intensity do not become zero but grow up to some extent caused by the diffraction. This is the first reason. In addition, light lays partially passes the liquid-crystal cells assigned to black. This is another reason.

Even if the top peaks of image-intensity curves were reduced by adjusting the transmittance of bright liquid-crystal cells of the L\&S patterns, the bottom peaks of the image-intensity curves are not decreased, or, the transmittance of black cells is always set at the minimum. Accordingly, the contrast of pattern images much degrades at the right bottom in the exposure field, where almost only the top peaks of image-intensity curves are greatly decreased.

The image contrast $C$ is defined by

$$
C=\frac{I_{\max }-I_{\min }}{I_{\max }+I_{\min }} \text {. }
$$

Here, $I_{\max }$ and $I_{\min }$ are the maximum and minimum values of the light intensity, respectively, as shown in Fig. 6 .

In addition, because the bottom peaks of intensity is kept almost steady, the mean intensity of exposure light in the lower right region is remained higher than that at the upper left corner. Therefore, the imbalance shown in Fig. 5 appears.

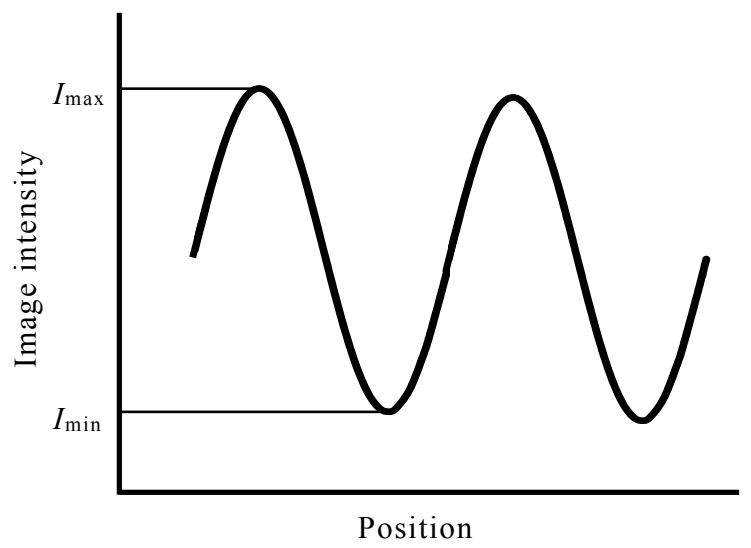

Fig. 6. Distribution of light-image intensity for L\&S patterns.
However, it is difficult to decrease the transmittance of the black cells, because it is always set at the minimum. For this reason, it was conceived an idea that the light intensity of black cells around the upper left corner, where the transparent light intensity of bright cells was low, was increased by contraries. When the transmittance of black cells around the upper left corner was decreased, the image contrast between the bright and black cells also decreased. Accordingly, the degraded image contrast around the upper left corner comes near the contrast of pattern images in the lower right region where the contrast was degraded by decreasing the transmittance of bright cells. Thus, by adopting the half-tone black cells, the image contrast was also balanced.

The changes of image-intensity curves at upper left corner and lower right are explained by Fig. 7. Fig. 7 (a) shows the initial image-intensity curves, and Fig. 7(b) shows the curves after only the top peaks in the lower right region were decreased. In this figure, imbalances of mean light intensity and image contrast are remained. If the bottom peaks of intensity were increased at the upper left corner, as shown in Fig. 7(c), however, the image contrast was also balanced.

Therefore, transmittances of both bright and black cells were adjusted. In addition, because the initial light intensity data were given by the intermittent contour map, as shown in Fig. 3, general gradation techniques were also applied. The modified transmittance distribution including all these compensations is shown in Fig. 8. Using this transmittance distribution, the test patterns were almost homogeneously printed in the exposure field, as shown in Fig. 9.

Next, pattern widths were measured at $8 \times 4=32$ divisions using an optical microscope (OLYMPUS, MX50). Measured pattern widths were shown in Fig. 10. In addition, 2and 4-pixel L\&S patterns in the 32 divisions are shown in Figs. 11 and 12 .

It was clarified that 4- and 5-pixel L\&S patterns with widths wider than approximately $37 \mu \mathrm{m}$ were almost homogeneously printed. The line-width errors were almost within $\pm 10 \%$ of the nominal widths. However, the pattern widths of 2- and 3-pixel L\&S patterns fluctuated slightly more 


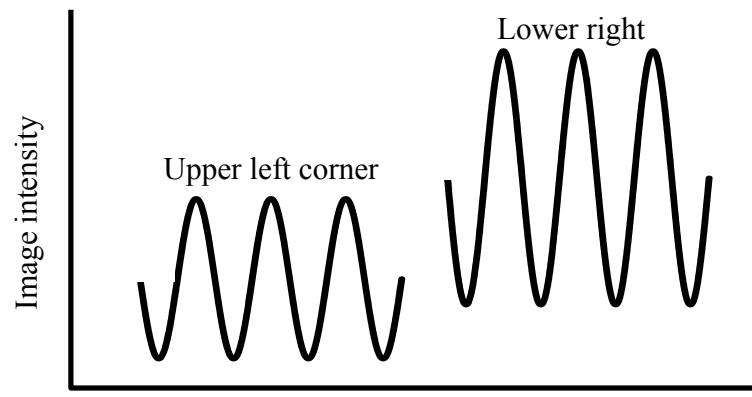

Position

(a) Initial difference of image intensity curves.

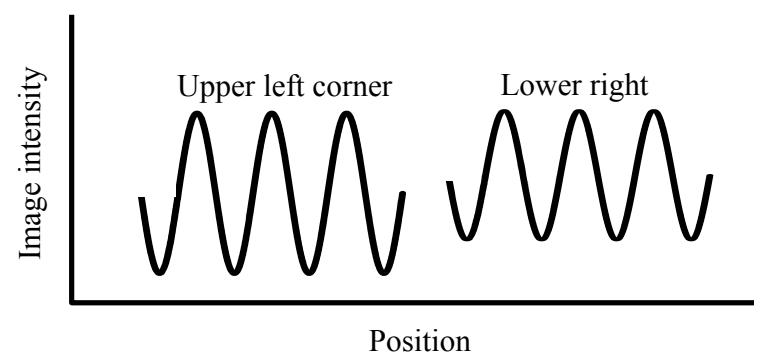

(b) After only the transmittances of bright cells are compensated.

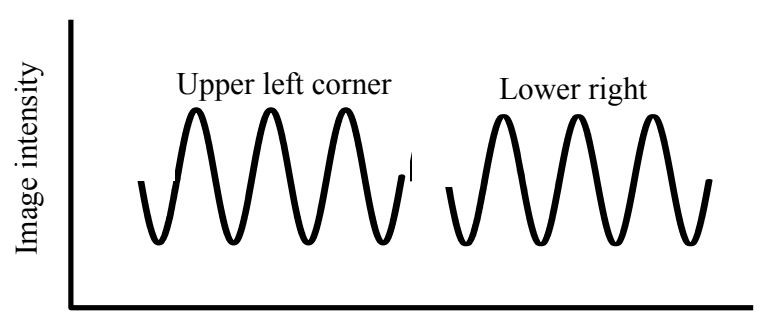

Position

(c) After the transmittances of black cells are also compensated.

Fig. 7. Explanation why the pattern width homogeneity was improved by adjusting both top peaks of bright-cell intensity and bottom peaks of black-cell intensity.

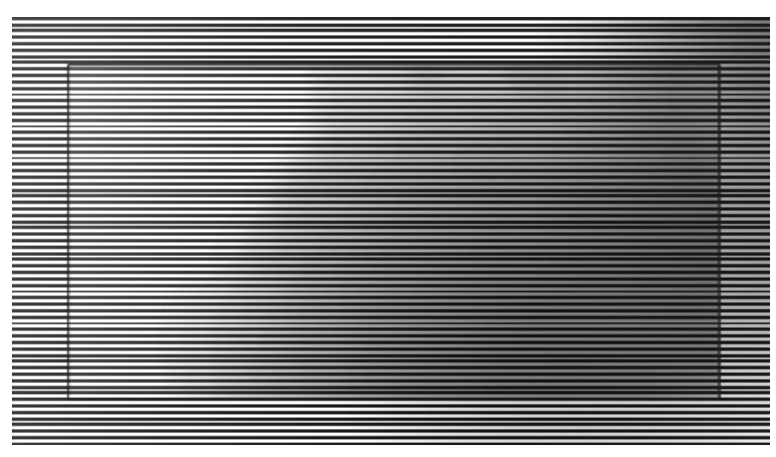

Fig. 8. Patterns designed for compensating both of the intensity and contrast distributions and superimposed on L\&S patterns.

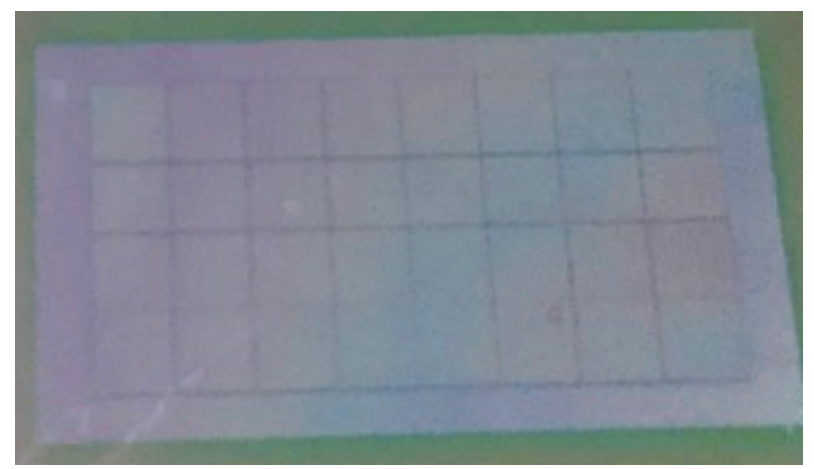

Fig. 9. 4-pixel L\&S patterns homogeneously printed in the exposure field by adjusting the transmittances of both bright and black cells.

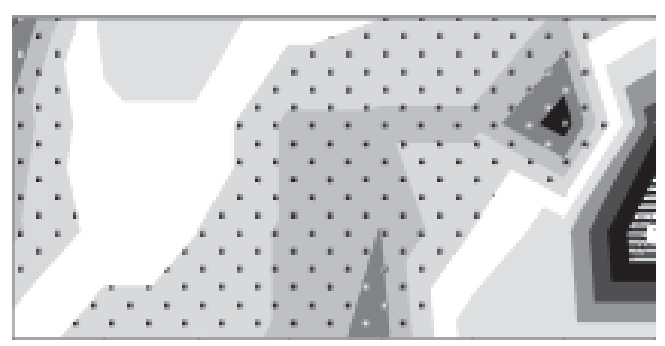

(a) 2-pixel L\&S patterns.

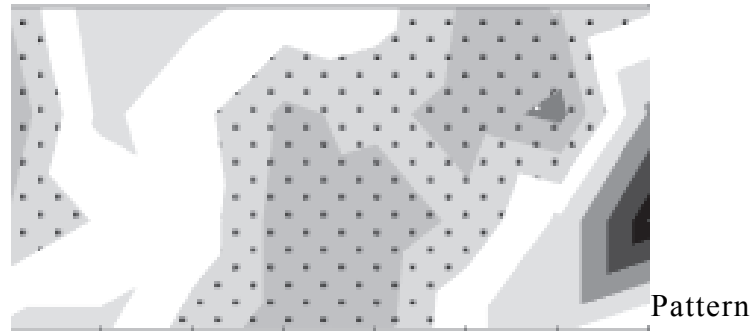

(b) 3-pixel L\&S patterns.

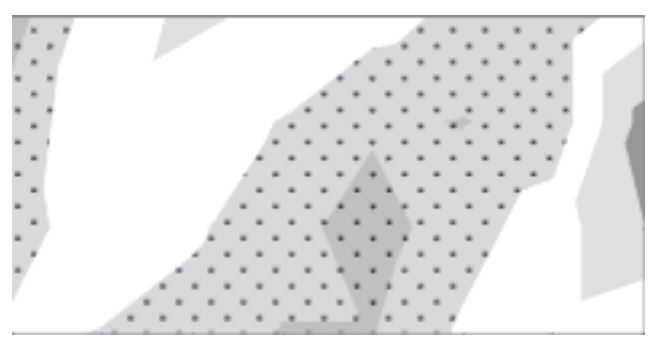

(c) 4-pixel L\&S patterns. width error $(\%)$

(d) 5-pixel L\&S patterns.

Fig. 10. Contour maps of measured pattern widths. 

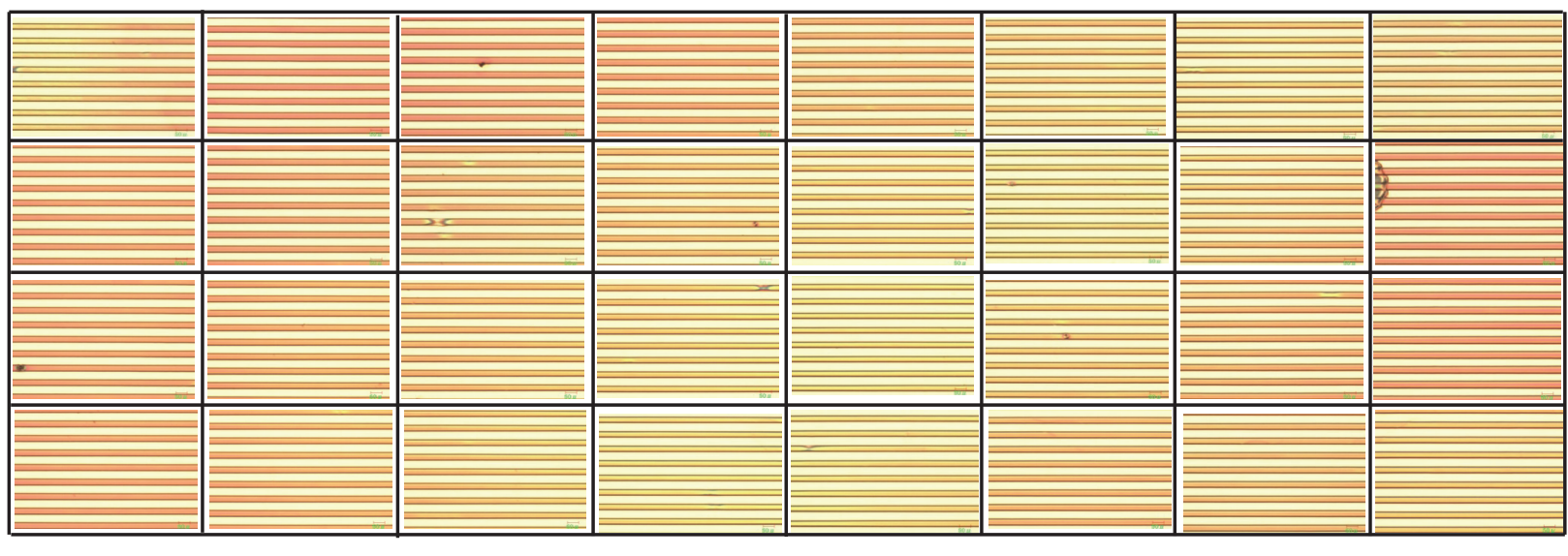

Fig. 11. Homogeneously printed 4-pixel L\&S patterns.

$100 \mu \mathrm{m}$
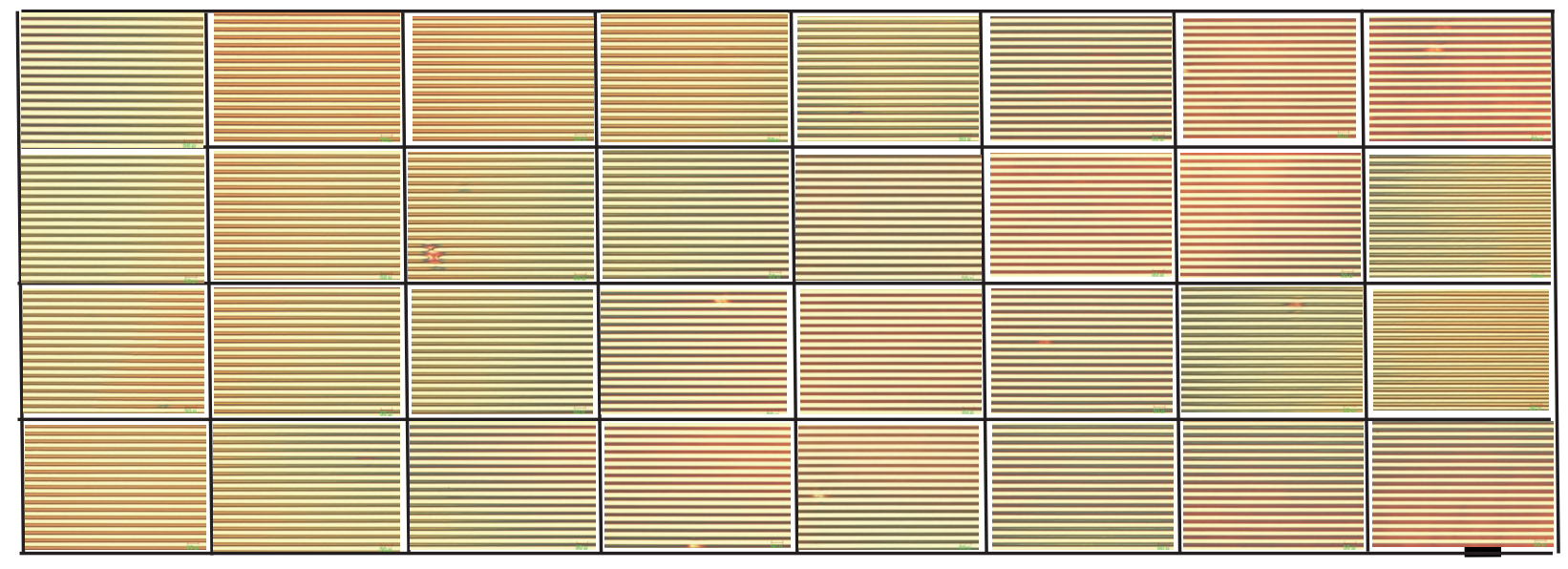

Fig. 12. Almost homogeneously printed 2-pixel L\&S patterns.

$100 \mu \mathrm{m}$

at several places in the exposure field. The pattern-width homogeneity should be improved further more.

\section{Discussion}

Prospects for further improvements and subjects hereafter are discussed. Owing to the new compensation measure, large distribution of the pattern widths was drastically improved. It was clarified that both modifications of light intensity and image contrast were necessary for homogenizing the pattern widths.

However, the intensity modification by decreasing the transmittance of bright cells loses the exposure light energy. In addition, the contrast modification by increasing the transmittance of black cells degrades the image contrast, that is, the absolute difference between the top and bottom peaks of the image intensity curves becomes small, and slopes of the image intensity curves become dull.
Accordingly, the compensation probably causes the decrease of exposure dose margin.

From this point of view, the original light intensity distribution in the exposure field should be suppressed as small as possible. The positions of the lamp source and projection lens should be adequately adjusted in the future.

On the other hand, to improve the pattern-width homogeneity immediately, the light intensity distribution should be grasped more precisely. It is necessary to express the light intensity distribution gradually and continuously, and the intensity and contrast have to be modified more smoothly. Transmittances of liquid-crystal cells should be modified by applying more carefully arranged gradations instead of using the step-like contour map of the light intensity and smoothing the steps by the versatile simple gradation.

In addition, it was difficult to measure the 
light intensity at the peripheries of the field accurately depending on the detector size this time. It is necessary to extend the modification in the whole exposure field including the periphery parts excluded this time.

\section{Conclusion}

Pattern width distribution in the exposure field of the desktop LCD matrix exposure system was drastically improved by applying the transmittance adjustments of both bright and black cells. In addition to the transmittance control of bright cells, the transmittances of black cells were also controlled for adjusting the image contrasts. As a result, 2-5 pixel L\&S patterns were almost homogeneously printed in the evaluation field. Because the pixel pitch was $9.3 \mu \mathrm{m}, 2-5$ pixels correspond to widths of 19-47 $\mu \mathrm{m}$. In particular, pattern-width errors were suppressed almost within $\pm 10 \%$ for 4 and 5-pixel L\&S patterns. It was clarified that not only the top peaks but also the bottom peaks of image intensity should be modified and the image contrast between the bright and black cells had to be appropriately adjusted.

\section{Acknowledgement}

This work was partially supported by Research Institute for Science and Technology of Tokyo Denki University, Grant Number Q15T-03.

\section{References}

1. K. Nakamura, H. Kubota, A. Nakada, T. Inokuchi, and K. Kosaka, Proc. SPIE, 4754 (2002) 737.
2. T. Morimoto, K. Nakamura, H. Kubota, A. Nakada, T. Akamichi, T. Inokuchi, and K. Kosaka, Proc. SPIE, 5130 (2003) 347.

3. K. Wakasugi, S. Wakimoto, T. Akamichi, A. Nakada, H. Kubota,T. Inokuchi, S. Suzuki, S. Aikawa, K. Kosaka, K. Nakamura, and T. Morimoto, IEEE Trans. Manufacturing, 18 (2005) 487.

4. T. Hayashi, T. Shibata, T. Kawashima, E. Makino, T. Mineta, and T. Masuzawa, Sensors and Actuators A, 144 (2008) 381.

5. M. V. Kessel and K. Heggarty, Microelectr. Eng., 86 (2009) 2385.

6. K. Itoga, J. Kobayashi, M. Yamato, and T. Okano, Methods in Cell Biol., 119 (2014) 141.

7. J. Cordeiro, M. Zelsmann, T. Honegger, E. Hadji. and D. Peyrade, Abs. $40^{\text {th }}$ Micro and Nano Eng., 2014 (MNE 2014) 31.

8. T. Horiuchi, T. Miyakawa, and S. Hosoda, Jpn. J. Appl. Phys., 39 (2000) 324.

9. T. Horiuchi and H. Kobayashi, Microelectronic Eng., 73-74 (2004) 48.

10. T. Horiuchi and H. Kobayashi, Jpn. J. Appl. Phys., 43 (2004) 394.

11. T. Horiuchi and A. Otani, Opt. Rev., 14 (2007) 224.

12. H. Fukasawa and T. Horiuchi, Jpn. J. Appl. Phys., 48 (2009) 082402.

13. T. Horiuchi, S. Siratori, Microelectr. Eng., 98 (2012) 574.

14. T. Horiuchi, and S. Siratori, J. Photopolym. Sci. Technol., 25 (2012) 455.

15. T. Horiuchi, S. Koyama, and H. Kobayashi, Microelectr. Eng., 141 (2015) 37. 